Supporting Information

for

\title{
Spectroscopic and Computational Studies of the ATP:corrinoid Adenosyltransferase (CobA) from Salmonella enterica: Insights into the Mechanism of Adenosylcobalamin Biosynthesis
}

Troy A. Stich, Nicole R. Buan, Jorge C. Escalante-Semerena, and Thomas C. Brunold

\section{Contents}

\section{Computational Details.}

Table S1-S2. Cartesian coordinates for computational models used in this study

Table S3. Fit parameters from Gaussian deconvolutions of AdoCbl + CobA/ATP spectra

Tables S4-S6. Selected $\mathrm{Co}^{2+} \mathrm{LF}$ transition energies $\left(\right.$ in $\mathrm{cm}^{-1}$ ) for all computational models employed in this study predicted by various methods

Tables S7-S8. Computed $g$-value and ${ }^{59} \mathrm{Co}$ hyperfine parameters for selected $\mathrm{Co}^{2+} \mathrm{Cbi}^{+}$models along the lower ligand bond dissociation pathway

Figure S1. Abs, CD, and MCD spectra of non-alkylated free $\mathrm{Co}^{3+}$ corriniods

Figures S2-S7. Abs, CD, and variable-temperature MCD spectra of all species explored in this report

Figure S8. Gaussian deconvoluted spectra of AdoCbl bound to CobA/ATP complex WEPR Input Files. 


\section{Computational Details}

DFT Calculations with ADF. The following two paragraphs (Slater Excitations and ZORA) provide the relevant details for the calculations of electronic transition energies and EPR parameters within the framework of density functional theory (DFT) as implemented in the Amsterdam Density Functional 2003.01 software package. ${ }^{1-3}$ All computations were performed on a cluster of 20 Intel Xeon processors using the Vosko-Wilk-Nusair local density approximation (VWN) ${ }^{4}$ along with the gradient corrections of Becke ${ }^{5}$ and Perdew ${ }^{6}$ for exchange and correlation, respectively. Atomic coordinates for the computational models used in these calculations were obtained as described in the Experimental section.

Slater Excitations. Electronic excitation energies for selected ligand field excited states were calculated using the Slater method by promoting half an electron from the donor MO to the acceptor MO and then taking the difference in MO energies upon SCF convergence. ${ }^{7}$

ZORA. Single point DFT calculations that make use of the zero-order regular approximation (ZORA) to the Dirac equation were performed to predict EPR parameters of the $\mathrm{Co}^{2+}$ corrinoid models. The relativistic set of triple- $\zeta$ Slater-type orbital (STO) ZORA basis set functions with polarization were used for all atoms. Molecular $g$-values were calculated with the spin-restricted, spin-orbit coupled ZORA (SO ROKS ZORA) Hamiltonian ${ }^{8}$ while the isotropic component to the nuclear hyperfine coupling constants for the ${ }^{59} \mathrm{Co}$ metal center and all coordinating ${ }^{14} \mathrm{~N}$ nuclei was computed using the scalar-relativistic, spin-unrestricted ZORA (SR UKS ZORA). ${ }^{9}$

DFT Calculations with ORCA. Electronic Abs and EPR spectroscopic properties were predicted by TD-DFT and CP-SCF DFT calculations, respectively, executed on a desktop PC using the ORCA 2.2 software package developed by Dr. Frank Neese (MPI Mülheim, Germany). ${ }^{10}$ The Perdew-Wang LDA (PW-LDA) ${ }^{11}$ in conjunction with the gradient corrections by Becke ${ }^{12}$ and Perdew ${ }^{6}$ were chosen for all DFT computations, as this combination yielded particularly accurate electronic structure descriptions in our earlier work on free $\mathrm{Co}^{2+}$ corrinoids. The DGauss (Gaussian polarized double valence orbital) ${ }^{13}$ basis set along with the Demon/J auxiliary basis ${ }^{14}$ were used on all atoms with the exception of the Co center that was treated with the TZVP triple- $\zeta$ basis. ${ }^{15}$

TD-DFT Calculations. Electronic excitation energies and intensities for all models were calculated by the TD-DFT method ${ }^{16-18}$ within the Tamm-Dancoff approximation ${ }^{19,20}$ as implemented in ORCA 2.2, using the same basis sets as described above for the single point calculations. At least 60 excited states were calculated by including all one-electron excitations within \pm 3 hartrees of the HOMO/LUMO energies, where the resolution of the identity approximation was used in calculating the Coulomb term to accelerate convergence of the TDDFT computations. ${ }^{21}$ Abs spectra predicted by the TD-DFT method were simulated assuming that each electronic transition gives rise to a Gaussian band with full width at half-maximum of $v_{1 / 2}=1250 \mathrm{~cm}^{-1}$ (chosen on the basis of our experimental data), in which case the molar 
extinction coefficient $\left(\varepsilon_{\text {max }}\right)$ is related to the predicted oscillator strength $\left(f_{\text {osc }}\right)$ by the formula $f_{\text {osc }}$ $=4.61 \times 10^{-9} \varepsilon_{\max } v_{1 / 2}{ }^{22}$

EPR Parameter Calculations. Molecular $g$ values and hyperfine parameters were calculated with the ORCA program by solving the coupled-perturbed SCF (CP-SCF) equations ${ }^{23}$ employing the PW-LDA functional. For each model considered, the "core-properties" 24 with extended polarization ${ }^{25}\left(\mathrm{CP}(\mathrm{PPP})\right.$ ) basis and Kutzelnigg's NMR/EPR (IGLOIII) basis ${ }^{26}$ were used to treat the cobalt center and all metal-ligating atoms ( $\mathrm{N}$ and $\mathrm{O})$, respectively, while the DGauss and Demon/J basis sets were used for all other atoms, as described for the single point calculations. The CP-SCF calculations of the $g$ values included all orbitals within a \pm 100 hartree window of the HOMO/LUMO energies with the origin of the g-"tensor" defined by the center of electronic charge of each model. A high-resolution radial grid with an integration accuracy of 7 was used and spin-orbit coupling contributions were included in the calculation of the ${ }^{59} \mathrm{Co}$ hyperfine tensor. ${ }^{27}$

INDO/S-CI. Ligand field transition energies for various low-spin $\mathrm{Co}^{2+}$ corrinoid and $\mathrm{Co}^{2+}$ corrinoid analogue models (Tables 3 and S4-S6) were computed using the semi-empirical INDO/S-CI approach of Zerner and co-workers ${ }^{28,29}$ as implemented in the ORCA 2.0 software package developed by Dr. Frank Neese (MPI Mülheim, Germany). The calculations employed the valence-shell ionization potentials and Slater-Condon parameters listed by Bacon and Zerner $^{30}$ and the standard interaction factors $f_{\mathrm{p} \sigma \mathrm{p} \sigma}=1.266$ and $f_{\mathrm{p} \pi \mathrm{p} \pi}=0.585$. Using the restricted open-shell Hartree-Fock SCF method, all calculations were converged tightly to the $S=1 / 2$ ground state that was then used as the reference state for a configuration interaction (CI) calculation that included all possible single electron excitations. Assignments for electronic transitions are based on the computed description of the excited states, which are given as linear combinations of one-electron excitations between ground state molecular orbitals.

The INDO/S-CI calculation of EPR properties of $\mathrm{Co}^{2+} \mathrm{Cbi}^{+}$models with differing $\mathrm{Co}-\mathrm{OH}_{2}$ bond lengths (Table S7) used the same complete active space for single excitations described above and included double electron excitations between the 20 highest-energy doubly occupied orbitals and the 18 lowest-energy virtual orbitals, which maintained the doublet multiplicity (i.e. CISD 0, 127, 46, 84, 0.5) of the ground state.

\section{References}

(1) te Velde, G.; Bickelhaupt, F. M.; van Gisbergen, S. J. A.; Guerra, C. F.; Baerends, E. J.; Snijders, J. G.; Ziegler, T. Journal of Computational Chemistry 2001, 22, 931-967.

(2) Guerra, C. F.; Snijders, J. G.; te Velde, G.; Baerends, E. J. Theor. Chem. Acc. 1998, 99, 391-403.

(3) ADF 2003.01, SCM, Theoretical Chemistry, Vrije Universiteit, Amsterdam, The Netherlands, http://www.scm.com

(4) Vosko, S. H.; Wilk, L.; Nusair, M. Can. J. Phys. 1980, 58, 1200-1211.

(5) Becke, A. D. Physical Review A 1988, 38, 3098-3100. 
(6) Perdew, J. P. Phys. Rev. B 1986, 33, 8822-8824.

(7) Slater, J. C. The Calculation of Molecular Orbitals; John Wiley \& Sons: New York, 1979.

(8) van Lenthe, E.; van der Avoird, A.; Wormer, P. E. S. Journal Chemical Physics 1997, 107, 2488-2498.

(9) van Lenthe, E.; van der Avoird, A.; Wormer, P. E. S. J. Chem. Phys. 1998, 108, 47834796.

(10) Neese, F.; Solomon, E. I. Inorg. Chem. 1999, 38, 1847-1865.

(11) Perdew, J. P.; Wang, Y. Phys. Rev. B 1992, 45, 13244-13249.

(12) Becke, A. D. J. Chem. Phys. 1986, 84, 4524-4529.

(13) Andzelm, J.; Wimmer, E. J. Chem. Phys. 1992, 96, 1280-1303.

(14) Godbout, N.; Salahub, D. R.; Andzeim, J.; Wimmer, E. Can. J. Chem. 1992, 70, 560-571.

(15) Schäfer, A.; Huber, C.; Ahlrichs, R. J. Chem. Phys. 1994, 100, 5829-5835.

(16) Bauernschmitt, R.; Ahlrichs, R. Chem. Phys. Lett. 1996, 256, 454-464.

(17) Casida, E. M.; Jamorski, C.; Casida, K. C.; Salahub, D. R. J. Chem. Phys. 1998, 108, 44394449.

(18) Stratmann, R. E.; Scuseria, G. E.; Frisch, M. J. J. Chem. Phys. 1998, 109, 8218-8224.

(19) Hirata, S.; Head-Gordon, M. Chem. Phys. Lett. 1999, 314, 291-299.

(20) Hirata, S.; Head-Gordon, M. Chem. Phys. Lett. 1999, 302, 375-382.

(21) Neese, F.; Olbrich, G. Chem. Phys. Lett. 2002, 362, 170-178.

(22) Lever, A. B. P. Inorganic Electronic Spectroscopy; 2nd ed.; Elsevier: Amsterdam ; New York, 1984.

(23) Neese, F. J. Chem. Phys. 2001, 115, 11080-11096.

(24) The ORCA basis set 'CoreProp' was used, which is based on the TurboMole DZ basis developed by Ahlrichs and coworkers. It can be obtained from the basis set library under ftp.chemie.uni-karlsruhe.de/pub/basen.

(25) The Ahlrichs ( $2 \mathrm{~d} 2 \mathrm{fg}, 3 \mathrm{p} 2 \mathrm{df}$ ) polarization functions were obtained from the TurboMole basis set library under ftp.chemie.uni-karlsruhe.de/pub/basen. Sc-Zn: $2 p$ functions were obtained from Wachters. J. Chem. Phys. 1970, 52, 1033, plus one f-function from the TurboMole library.

(26) Kutzelnigg, W.; Fleischer, U.; Schindler, M. The IGLO Method: Ab Initio Calculation and Interpretation of NMR Chemical Shifts and Magnetic Susceptibilities; Springer-Verlag: Heidelberg, 1990; Vol. 23.

(27) Neese, F. J. Chem. Phys. 2003, 118, 3939-3948.

(28) Ridley, J.; Zerner, M. C. Theor. Chim. Acta 1973, 32, 111-134.

(29) Zerner, M. C.; Loew, G. H.; Kirchner, R. F.; Mueller-Westerhof, U. T. J. Am. Chem. Soc. 1980, 102, 589-599.

(30) Bacon, A. D.; Zerner, M. C. Theor. Chim. Acta 1979, 53, 21-54. 
Table S1. Cartesian Coordinates for $\mathrm{Co}^{2+} \mathrm{Cbi}^{+}$Computational Model

\begin{tabular}{lrrr}
\hline Atom & $\mathrm{x}(\AA)$ & $\mathrm{y}(\AA)$ & $\mathrm{z}(\AA)$ \\
\hline $\mathrm{Co}$ & 0.000000 & 0.000000 & 0.000000 \\
$\mathrm{C}$ & -2.456116 & -1.415939 & -0.187225 \\
$\mathrm{C}$ & -3.945496 & -1.242859 & 0.425919 \\
$\mathrm{C}$ & -4.152008 & 0.260437 & 0.257034 \\
$\mathrm{C}$ & -2.748413 & 0.818954 & 0.344620 \\
$\mathrm{C}$ & -2.429230 & 2.168365 & 0.692535 \\
$\mathrm{C}$ & -1.180283 & 2.682953 & 0.562347 \\
$\mathrm{C}$ & -0.698685 & 4.110336 & 0.813950 \\
$\mathrm{C}$ & 0.618500 & 4.109528 & -0.000519 \\
$\mathrm{C}$ & 1.007828 & 2.668625 & 0.060516 \\
$\mathrm{C}$ & 2.285309 & 2.265869 & 0.015152 \\
$\mathrm{C}$ & 2.747269 & 0.934341 & 0.050598 \\
$\mathrm{C}$ & 4.156479 & 0.553223 & 0.186325 \\
$\mathrm{C}$ & 4.109344 & -0.960358 & -0.208130 \\
$\mathrm{C}$ & 2.654907 & -1.282700 & 0.062500 \\
$\mathrm{C}$ & 2.168594 & -2.588593 & 0.215347 \\
$\mathrm{C}$ & 0.744461 & -2.849411 & 0.298584 \\
$\mathrm{C}$ & 0.096909 & -4.188721 & 0.600388 \\
$\mathrm{C}$ & -1.361267 & -3.884537 & 0.177139 \\
$\mathrm{C}$ & -1.482208 & -2.353745 & 0.549271 \\
$\mathrm{~N}$ & -1.868622 & -0.093857 & 0.045792 \\
$\mathrm{~N}$ & -0.086334 & 1.899765 & 0.192795 \\
$\mathrm{~N}$ & 1.890213 & -0.094100 & 0.046539 \\
$\mathrm{~N}$ & -0.128464 & -1.858780 & 0.192123 \\
$\mathrm{O}$ & 0.036953 & 0.012249 & -2.201109 \\
$\mathrm{H}$ & -2.477951 & -1.669998 & -1.236725 \\
$\mathrm{H}$ & -4.703522 & -1.862503 & -0.029907 \\
$\mathrm{H}$ & -3.931488 & -1.478378 & 1.479874 \\
$\mathrm{H}$ & -4.623596 & 0.653763 & -0.631439 \\
$\mathrm{H}$ & -4.883072 & 0.556900 & 0.995697 \\
$\mathrm{H}$ & -3.250427 & 2.792023 & 1.013763 \\
$\mathrm{H}$ & -1.374512 & 4.902512 & 0.527573 \\
$\mathrm{H}$ & -0.467422 & 4.165741 & 1.867493 \\
$\mathrm{H}$ & 0.452942 & 4.457794 & -1.009384 \\
$\mathrm{H}$ & 1.354721 & 4.808685 & 0.378006 \\
$\mathrm{H}$ & 3.040558 & 3.032547 & -0.046890 \\
$\mathrm{H}$ & 4.385986 & 0.598633 & 1.240768 \\
$\mathrm{H}$ & 4.861496 & 1.202271 & -0.311707 \\
$\mathrm{H}$ & 4.301071 & -1.198608 & -1.243988 \\
$\mathrm{H}$ & 4.865219 & -1.527542 & 0.315002 \\
$\mathrm{H}$ & 2.875885 & -3.401474 & 0.288193 \\
$\mathrm{H}$ & 0.147934 & -4.435791 & 1.650467 \\
$\mathrm{H}$ & 0.466003 & -5.016708 & 0.013275 \\
$\mathrm{H}$ & -2.140656 & -4.460587 & 0.653717 \\
$\mathrm{H}$ & -1.500916 & -4.161285 & -0.858521 \\
$\mathrm{H}$ & -1.241806 & -2.308487 & 1.601181 \\
$\mathrm{H}$ & -0.285519 & 0.759477 & -2.767752 \\
$\mathrm{H}$ & 0.401071 & -0.725210 & -2.751158 \\
\hline & & &
\end{tabular}


Table S2. Cartesian Coordinates for $\left[\mathrm{Co}\left(\mathrm{Me}_{6}[14] \mathrm{dieneN}_{4}\right)\left(\mathrm{H}_{2} \mathrm{O}\right)\right]$ Computational Model

\begin{tabular}{lrrr}
\hline Atom & $\mathrm{x}(\AA)$ & $\mathrm{y}(\AA)$ & $\mathrm{z}(\AA)$ \\
\hline $\mathrm{Co}$ & 0.000000 & 0.000000 & 0.000000 \\
$\mathrm{~N}$ & 0.031021 & 1.971252 & 0.119186 \\
$\mathrm{C}$ & -1.309600 & 2.373596 & 0.186737 \\
$\mathrm{C}$ & -2.278442 & 1.377304 & -0.428879 \\
$\mathrm{~N}$ & -1.916321 & 0.060410 & 0.010773 \\
$\mathrm{C}$ & -2.851013 & -0.986176 & 0.193802 \\
$\mathrm{C}$ & -4.238464 & -0.686935 & 0.094223 \\
$\mathrm{C}$ & -2.397232 & -2.190659 & 0.493591 \\
$\mathrm{C}$ & -1.105331 & -2.453186 & 1.090271 \\
$\mathrm{C}$ & -0.831909 & -1.823151 & 2.456345 \\
$\mathrm{C}$ & -0.975876 & -3.982056 & 1.300156 \\
$\mathrm{~N}$ & 0.031021 & -1.945953 & 0.121109 \\
$\mathrm{C}$ & 1.401794 & -2.453796 & 0.438080 \\
$\mathrm{C}$ & 2.259491 & -1.545944 & -0.148285 \\
$\mathrm{~N}$ & 1.830841 & -0.096024 & 0.006409 \\
$\mathrm{C}$ & 2.680450 & 0.801605 & 0.170242 \\
$\mathrm{C}$ & 4.254105 & 0.807983 & 0.042236 \\
$\mathrm{C}$ & 2.476563 & 2.179123 & 0.462540 \\
$\mathrm{C}$ & 0.990768 & 2.539474 & 0.997864 \\
$\mathrm{C}$ & 0.889465 & 1.996231 & 2.334244 \\
$\mathrm{C}$ & 0.968292 & 3.959610 & 0.997009 \\
$\mathrm{H}$ & 0.444321 & 2.379715 & -0.706894 \\
$\mathrm{H}$ & -1.562958 & 2.504807 & 1.228363 \\
$\mathrm{H}$ & -1.393967 & 3.316910 & -0.332336 \\
$\mathrm{H}$ & -2.235229 & 1.451141 & -1.505478 \\
$\mathrm{H}$ & -3.279663 & 1.607635 & -0.095886 \\
$\mathrm{H}$ & -4.381836 & 0.352371 & -0.162048 \\
$\mathrm{H}$ & -4.704590 & -0.877914 & 1.049545 \\
$\mathrm{H}$ & -4.677292 & -1.316330 & -0.665817 \\
$\mathrm{H}$ & -3.115372 & -2.596924 & 1.190430 \\
$\mathrm{H}$ & -2.428848 & -2.754608 & -0.426910 \\
$\mathrm{H}$ & -0.913315 & -0.748367 & 2.388290 \\
$\mathrm{H}$ & 0.169174 & -2.082382 & 2.767822 \\
$\mathrm{H}$ & -1.539932 & -2.200974 & 3.179077 \\
$\mathrm{H}$ & -1.022919 & -0.017044 & -2.315247 \\
$\mathrm{H}$ & -0.010056 & -4.198898 & 1.732100 \\
$\mathrm{H}$ & -1.058044 & -4.479660 & 0.345139 \\
$\mathrm{H}$ & -1.754883 & -4.350677 & 1.951035 \\
$\mathrm{H}$ & -0.180634 & -2.354416 & -0.778015 \\
$\mathrm{H}$ & -0.798105 & -0.094600 & -2.903924 \\
\hline $\mathrm{H}$ & 1.546616 & -3.433929 & 0.008240 \\
$\mathrm{H}$ & 1.549316 & -2.493332 & 1.507217 \\
$\mathrm{H}$ & 3.232208 & -1.667816 & 0.304855 \\
$\mathrm{H}$ & 2.326324 & -1.763031 & -1.204117 \\
$\mathrm{H}$ & 4.592712 & -0.189957 & -0.194046 \\
$\mathrm{H}$ & 4.682327 & 1.122894 & 0.982361 \\
$\mathrm{H}$ & 4.565552 & 1.491379 & -0.733887 \\
$\mathrm{H}$ & 3.182724 & 2.458405 & 1.230469 \\
$\mathrm{H}$ & 2.661880 & 2.756332 & -0.431244 \\
$\mathrm{H}$ & 1.633087 & 2.463074 & 2.963104 \\
$\mathrm{H}$ & 1.057526 & 0.929520 & 2.316391 \\
$\mathrm{H}$ & -0.0077602 & 2.213089 & 2.733521 \\
$\mathrm{H}$ & 4.356537 & 1.671722 \\
$\mathrm{H}$ & & & \\
$\mathrm{H}$ & & &
\end{tabular}


Table S3. Fit Parameters from Gaussian Deconvolution of the Abs, CD, and MCD spectra of AdoCbl + CobA/ATP (Figure S8)

\begin{tabular}{llccc}
\hline Band & $\begin{array}{l}\text { Energy } \\
\left(\mathrm{cm}^{-1}\right)\end{array}$ & $\begin{array}{l}\varepsilon(\mathrm{Abs}) \\
\left(\mathrm{mM}^{-1} \mathrm{~cm}^{-1}\right)\end{array}$ & $\begin{array}{l}\Delta \varepsilon(\mathrm{CD}) \\
\left(\mathrm{M}^{-1} \mathrm{~cm}^{-1}\right)\end{array}$ & $\begin{array}{l}\Delta \varepsilon(\mathrm{MCD}) \\
\left(\mathrm{M}^{-1} \mathrm{~cm}^{-1}\right)\end{array}$ \\
\hline 1 & 17950 & 6.8 & -14.8 & 12.5 \\
2 & 19000 & 10.2 & -9.8 & 5.9 \\
3 & 20600 & 5.2 & 8.8 & -12.5 \\
4 & 21820 & 3.3 & 7.0 & -8.8 \\
5 & 23300 & 4.3 & -5.0 & +0.9 \\
6 & 24900 & 3.7 & -0.1 & 4.2 \\
7 & 26400 & 14.2 & 10.0 & 17.6 \\
8 & 27800 & 11.0 & -14.0 & 11.7 \\
9 & 28900 & 7.7 & -13.0 & -6.4 \\
10 & 29800 & 11.5 & 1.4 & -2.6 \\
11 & 31400 & 16.0 & -9.4 & 5.5 \\
\hline
\end{tabular}

Table S4. Selected $\mathrm{Co}^{2+} \mathrm{Cbl} \mathrm{LF}$ Transition Energies (in $\mathrm{cm}^{-1}$ ) Predicted by Various Computational Methods

\begin{tabular}{|c|c|c|c|c|c|c|c|}
\hline & $\begin{array}{c}\text { INDO/S-CI CIS } \\
(0,145,0.5) \\
\end{array}$ & & $\begin{array}{l}\text { TD-DFT } \\
\text { B3LYP } \\
\end{array}$ & & $\begin{array}{c}\text { TD-DFT } \\
\text { BP86 } \\
\end{array}$ & & Slater $1 / 2 \mathrm{e}^{-}$Excitations \\
\hline Energy & & Energy & & Energy & & Energy & \\
\hline 4409 & $\mathrm{~d}_{\mathrm{yz}} \rightarrow \mathrm{d}_{\mathrm{z}^{2}}$ & 11045 & $\mathrm{~d}_{\mathrm{yz}} \rightarrow \mathrm{d}_{\mathrm{z}^{2}}$ & 14090 & $\mathrm{~d}_{\mathrm{yz}} \rightarrow \mathrm{d}_{\mathrm{z}^{2}}$ & 9638 & $\beta \mathrm{d}_{\mathrm{yz}} \rightarrow \mathrm{d}_{\mathrm{z}^{2}}$ \\
\hline 5075 & $\mathrm{~d}_{\mathrm{xz}} \rightarrow \mathrm{d}_{\mathrm{z}^{2}}$ & 12240 & $\mathrm{~d}_{\mathrm{xz}} \rightarrow \mathrm{d}_{\mathrm{z}^{2}}$ & 15910 & $\mathrm{~d}_{\mathrm{xz}} \rightarrow \mathrm{d}_{\mathrm{z}^{2}}$ & 11275 & $\beta \mathrm{d}_{\mathrm{xz}} \rightarrow \mathrm{d}_{\mathrm{z}^{2}}$ \\
\hline 13257 & 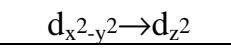 & 17185 & $\mathrm{~d}_{\mathrm{yz}} \rightarrow \mathrm{d}_{\mathrm{xy}}$ & 20000 & $\mathrm{~d}_{\mathrm{z}} 2 \rightarrow \mathrm{d}_{\mathrm{xy}}$ & 17446 & $\beta \mathrm{d}_{\mathrm{x}^{2}-\mathrm{y}^{2} \rightarrow \mathrm{d}_{\mathrm{z}^{2}}}$ \\
\hline
\end{tabular}

Table S5. Selected $\mathrm{Co}^{2+} \mathrm{Cbi}^{+} \mathrm{LF}$ Transition Energies (in $\mathrm{cm}^{-1}$ ) Predicted by Various Computational Methods

\begin{tabular}{|c|c|c|c|c|c|c|c|}
\hline & $\begin{array}{c}\text { INDO/S-CI CIS } \\
(0,127,0.5)\end{array}$ & & $\begin{array}{l}\text { TD-DFT } \\
\text { B3LYP }\end{array}$ & & $\begin{array}{l}\text { TD-DFT } \\
\text { BP86 }\end{array}$ & & Slater $1 / 2 \mathrm{e}^{-}$Excitations \\
\hline Energy & & Energy & & Energy & & Energy & \\
\hline 3543 & $\mathrm{~d}_{\mathrm{yz}} \rightarrow \mathrm{d}_{\mathrm{z}^{2}}$ & 8487 & $\mathrm{~d}_{\mathrm{yz}} \rightarrow \mathrm{d}_{\mathrm{z}^{2}}$ & 11330 & $\mathrm{~d}_{\mathrm{yz}} \rightarrow \mathrm{d}_{\mathrm{z}^{2}}$ & 6033 & $\beta \mathrm{d}_{\mathrm{yz}} \rightarrow \mathrm{d}_{\mathrm{z}^{2}}$ \\
\hline 3826 & $\mathrm{~d}_{\mathrm{xz}} \rightarrow \mathrm{d}_{\mathrm{z}^{2}}$ & 9267 & $\mathrm{~d}_{\mathrm{xz}} \rightarrow \mathrm{d}_{\mathrm{z}^{2}}$ & 12975 & $\mathrm{~d}_{\mathrm{xz}} \rightarrow \mathrm{d}_{\mathrm{z}^{2}}$ & 7146 & $\beta \mathrm{d}_{\mathrm{xz}} \rightarrow \mathrm{d}_{\mathrm{z}^{2}}$ \\
\hline 13792 & 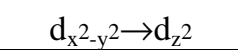 & 16333 & 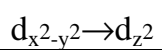 & 20565 & $\mathrm{~d}_{\mathrm{x}^{2}-\mathrm{y}^{2} \rightarrow \mathrm{d}^{2}}$ & 13630 & 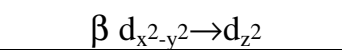 \\
\hline
\end{tabular}


Table S6. Selected $\left[\mathrm{Co}\left(\mathrm{Me}_{6}[14]\right.\right.$ dieneN $\left.\left._{4}\right)\left(\mathrm{H}_{2} \mathrm{O}\right)\right]$ LF Transition Energies (in $\mathrm{cm}^{-1}$ ) Predicted by Various Computational Methods

\begin{tabular}{lccccccc}
\hline & $\begin{array}{c}\text { INDO/S-CI CIS } \\
(1,125,0.5)\end{array}$ & \multicolumn{3}{c}{ TD-DFT } & B3LYP & TD-DFT \\
& & EP866 & \multicolumn{3}{c}{ Slater $1 / 2 \mathrm{e}^{-}$excitation } \\
\hline Energy & & & Energy & \multicolumn{3}{c}{ Energy } \\
3119 & $\mathrm{~d}_{\mathrm{yz}} \rightarrow \mathrm{d}_{\mathrm{z}^{2}}$ & 7750 & $\mathrm{~d}_{\mathrm{yz}} \rightarrow \mathrm{d}_{\mathrm{z}^{2}}$ & 12300 & $\mathrm{~d}_{\mathrm{xz}} \rightarrow \mathrm{d}_{\mathrm{z}^{2}}$ & 4875 & $\beta \mathrm{d}_{\mathrm{yz}} \rightarrow \mathrm{d}_{\mathrm{z}^{2}}$ \\
3556 & $\mathrm{~d}_{\mathrm{xz}} \rightarrow \mathrm{d}_{\mathrm{z}^{2}}$ & 8850 & $\mathrm{~d}_{\mathrm{xz}} \rightarrow \mathrm{d}_{\mathrm{z}^{2}}$ & 13125 & $\mathrm{~d}_{\mathrm{yz}} \rightarrow \mathrm{d}_{\mathrm{z}^{2}}$ & 5125 & $\beta \mathrm{d}_{\mathrm{xz}} \rightarrow \mathrm{d}_{\mathrm{z}^{2}}$ \\
11502 & $\mathrm{~d}_{\mathrm{xy}} \rightarrow \mathrm{d}_{\mathrm{z}^{2}}$ & 15575 & $\mathrm{~d}_{\mathrm{xy}} \rightarrow \mathrm{d}_{\mathrm{z}^{2}}$ & 20725 & $\mathrm{~d}_{\mathrm{xy}} \rightarrow \mathrm{d}_{\mathrm{z}^{2}}$ & 12975 & $\beta \mathrm{d}_{\mathrm{xy}} \rightarrow \mathrm{d}_{\mathrm{z}^{2}}$ \\
\hline
\end{tabular}

Table S7. INDO/S-CI $g$-value Calculations for Selected $\mathrm{Co}^{2+} \mathrm{Cbi}^{+}$Models along the Lower Ligand Dissociation Pathway Computed using the Complete Active Space for Single Excitations and a Large Window for Double Electron Excitations, CISD (0, 127, 46, 84, 0.5)

\begin{tabular}{l|ccc}
\hline $\mathbf{r}_{\mathrm{Co}-\mathrm{OH}}$ & $g_{\mathrm{z}}$ & $g_{\mathrm{y}}$ & $g_{\mathrm{x}}$ \\
\hline 2.100 & 2.014 & 2.184 & 2.200 \\
2.201 & 2.014 & 2.193 & 2.209 \\
2.250 & 2.014 & 2.196 & 2.212 \\
2.300 & 2.014 & 2.200 & 2.215 \\
2.350 & 2.014 & 2.203 & 2.218 \\
2.450 & 2.013 & 2.208 & 2.223 \\
2.550 & 2.013 & 2.211 & 2.226 \\
2.650 & 2.013 & 2.214 & 2.229 \\
\hline
\end{tabular}

Table S8. Pure DFT (PW-LDA) $g$-value and ${ }^{59}$ Co Hyperfine Parameters (in MHz) Computed for Selected $\mathrm{Co}^{2+} \mathrm{Cbi}^{+}$Models along the Lower Ligand Dissociation Pathway

\begin{tabular}{l|cccrrr}
\hline $\mathbf{r}_{\mathrm{Co}-\mathrm{OH}}$ & $g_{\mathrm{z}}$ & $g_{\mathrm{y}}$ & $g_{\mathrm{x}}$ & $A_{\mathrm{z}}(\mathrm{Co})$ & $A_{\mathrm{y}}(\mathrm{Co})$ & $A_{\mathrm{x}}(\mathrm{Co})$ \\
\hline 2.100 & 2.004 & 2.092 & 2.101 & 538 & 130 & 82 \\
2.201 & 2.004 & 2.098 & 2.108 & 575 & 173 & 131 \\
2.250 & 2.004 & 2.101 & 2.112 & 589 & 192 & 152 \\
2.300 & 2.004 & 2.104 & 2.115 & 604 & 209 & 172 \\
2.350 & 2.004 & 2.106 & 2.118 & 617 & 225 & 190 \\
2.450 & 2.005 & 2.111 & 2.123 & 639 & 253 & 220 \\
2.550 & 2.005 & 2.114 & 2.128 & 657 & 275 & 246 \\
2.650 & 2.005 & 2.117 & 2.132 & 672 & 294 & 267 \\
\hline
\end{tabular}




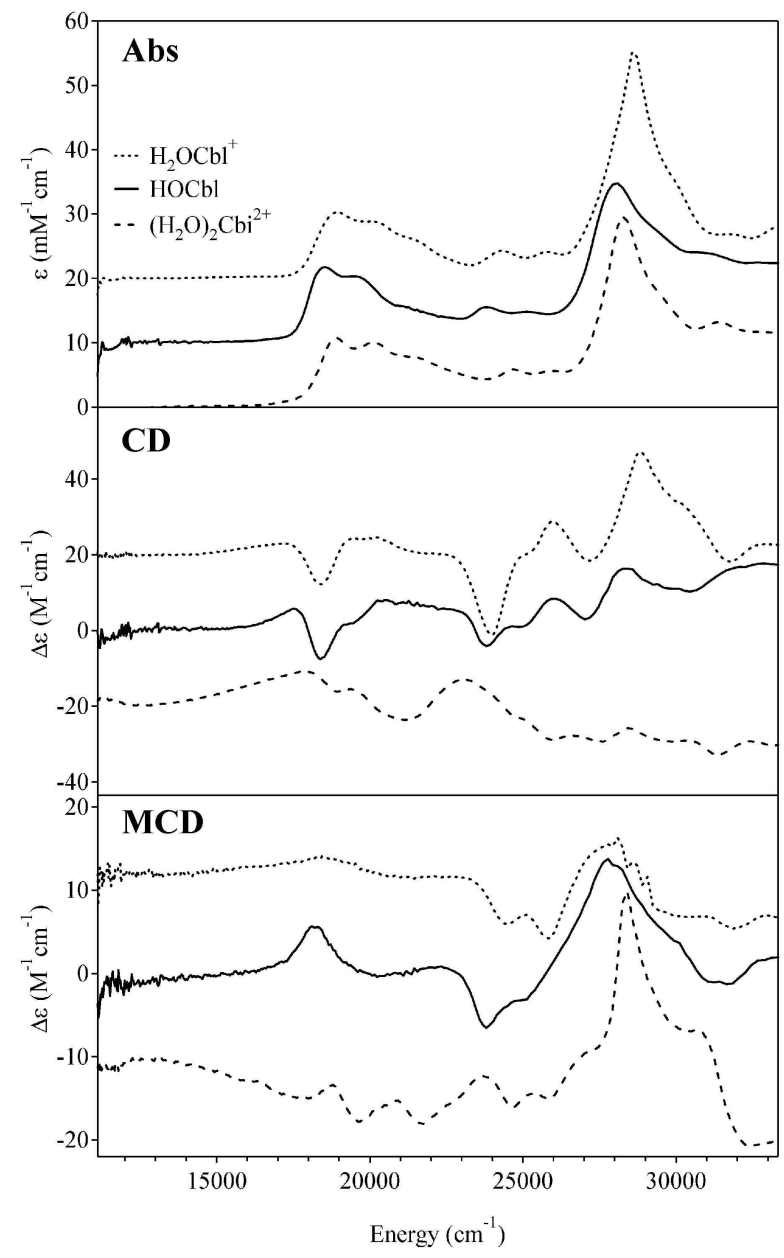

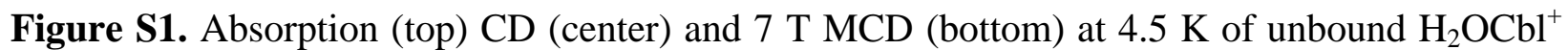
(pH 5), $\mathrm{HOCbl}(\mathrm{pH} 9)$, and $\left(\mathrm{H}_{2} \mathrm{O}\right)_{2} \mathrm{Cbl}^{2+}$. 


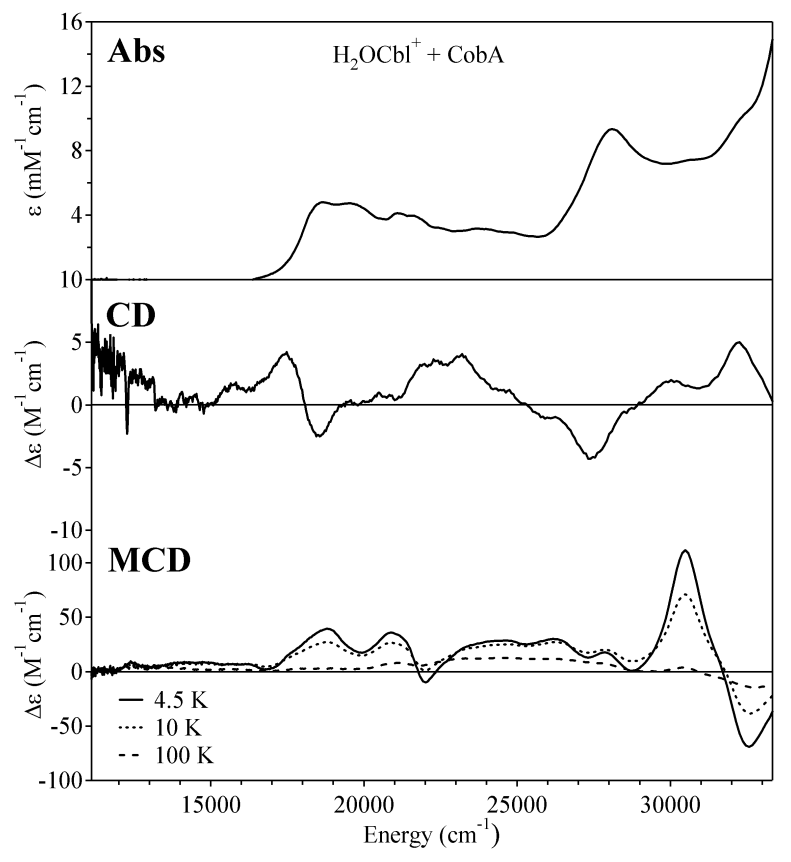

Figure S2. Absorption (top) and $\mathrm{CD}$ (center) spectra recorded at $4.5 \mathrm{~K}$ and $7 \mathrm{~T}$ variable temperature MCD (bottom) spectra of $\mathrm{H}_{2} \mathrm{OCbl}^{+}$in the presence of CobA.

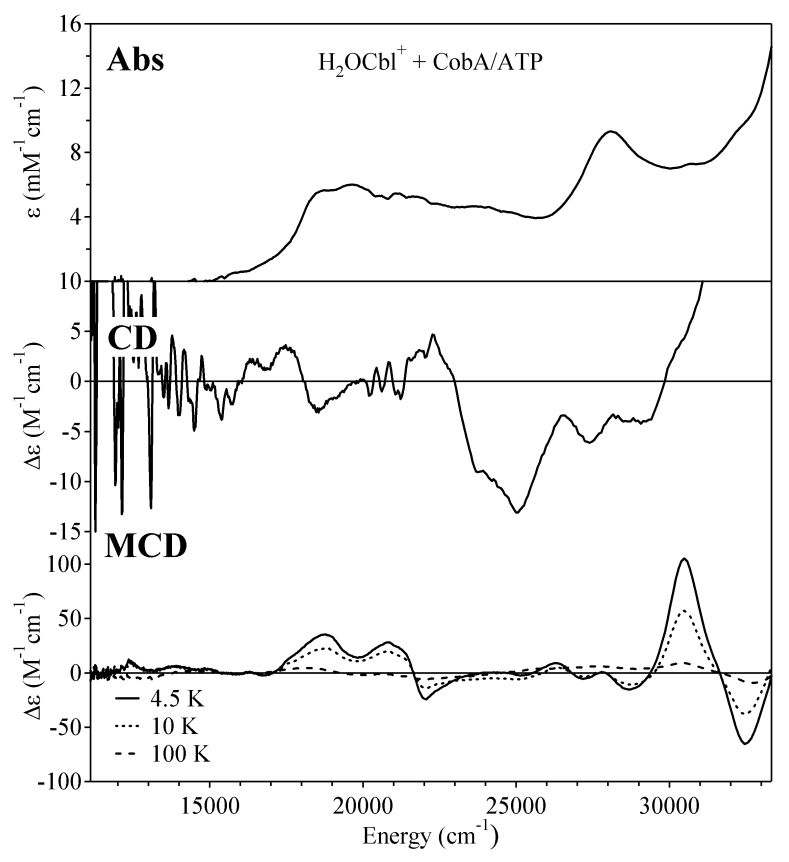

Figure S3. Absorption (top) and $\mathrm{CD}$ (center) spectra recorded at $4.5 \mathrm{~K}$ and $7 \mathrm{~T}$ variable temperature $\mathrm{MCD}$ (bottom) spectra of $\mathrm{H}_{2} \mathrm{OCbl}^{+}$in the presence of $\mathrm{CobA} / \mathrm{ATP}$ complex. 


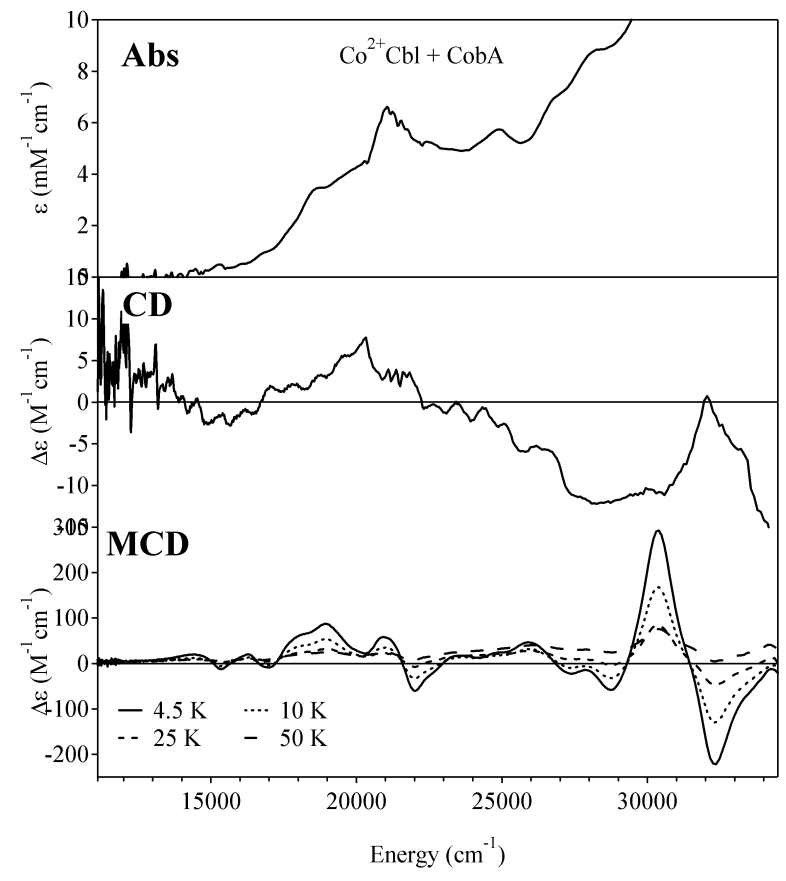

Figure S4. Absorption (top) and $\mathrm{CD}$ (center) spectra recorded at $4.5 \mathrm{~K}$ and $7 \mathrm{~T}$ variable temperature MCD (bottom) spectra of $\mathrm{Co}^{2+} \mathrm{Cbl}$ in the presence of CobA.

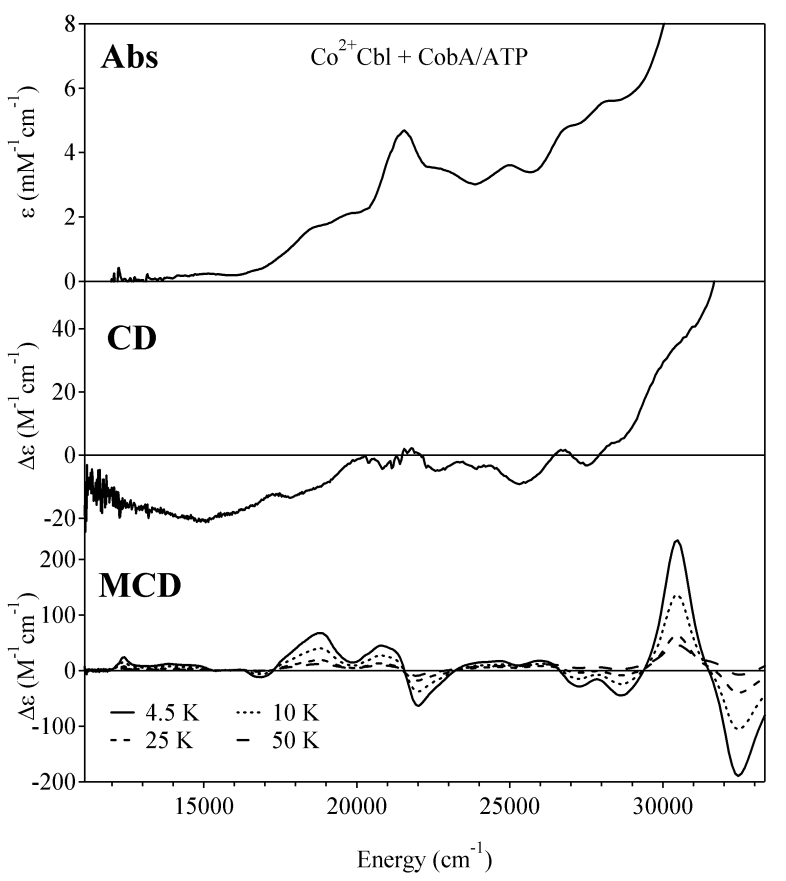

Figure S5. Absorption (top) and CD (center) spectra recorded at $4.5 \mathrm{~K}$ and $7 \mathrm{~T}$ variable temperature MCD (bottom) spectra of $\mathrm{Co}^{2+} \mathrm{Cbl}$ in the presence of $\mathrm{CobA} / \mathrm{ATP}$ complex. 


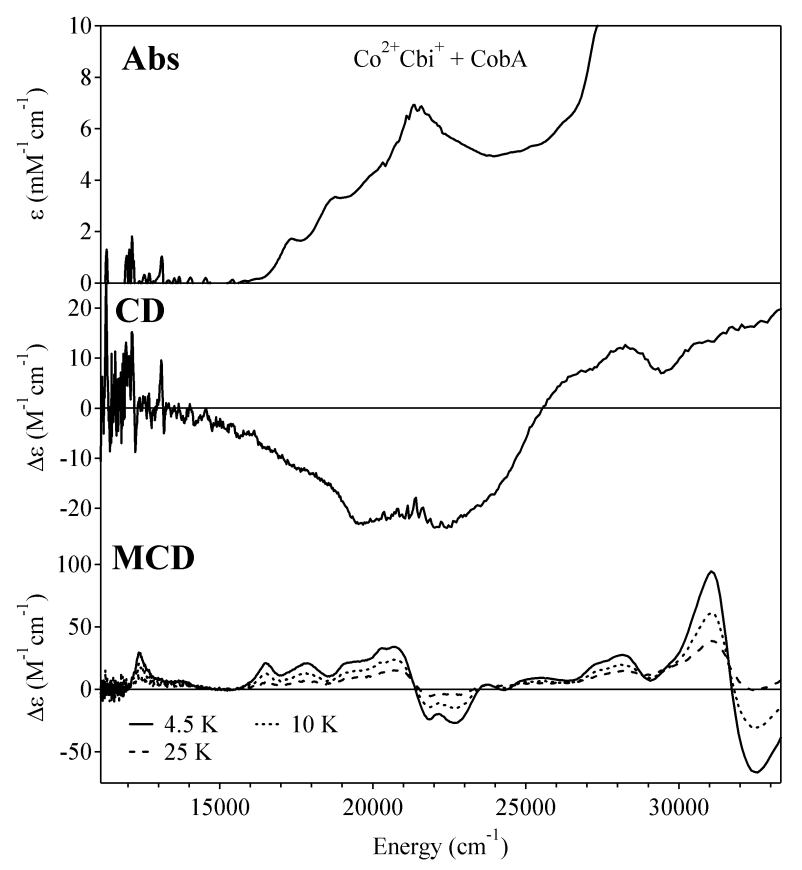

Figure S6. Absorption (top) and $\mathrm{CD}$ (center) spectra recorded at $4.5 \mathrm{~K}$ and $7 \mathrm{~T}$ variable temperature MCD (bottom) spectra of $\mathrm{Co}^{2+} \mathrm{Cbi}^{+}$in the presence of CobA.

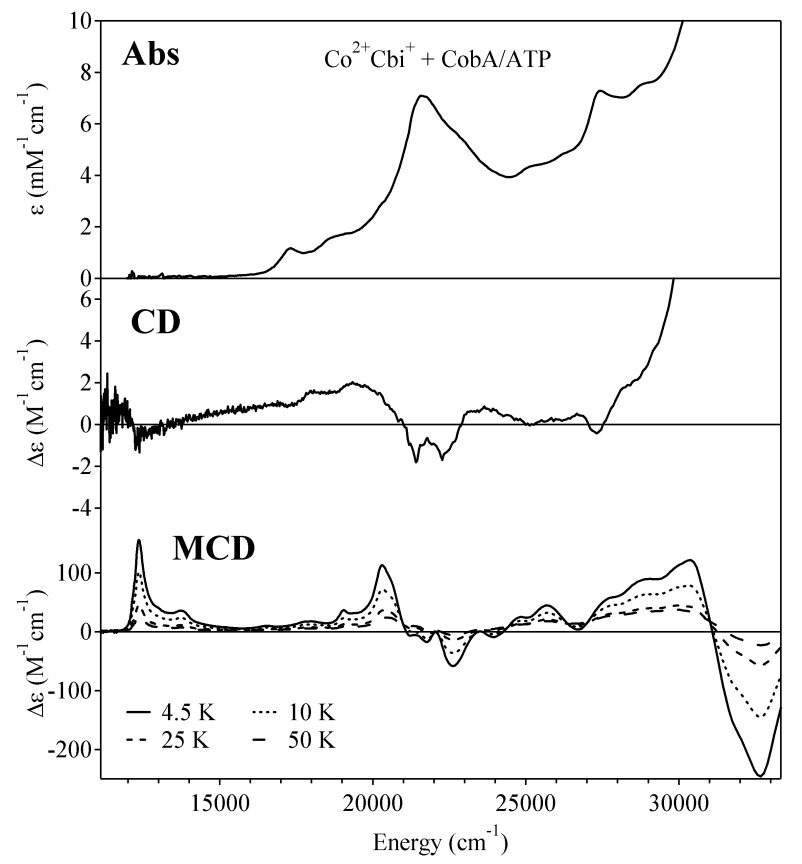

Figure S7. Absorption (top) and CD (center) spectra recorded at $4.5 \mathrm{~K}$ and $7 \mathrm{~T}$ variable temperature MCD (bottom) spectra of $\mathrm{Co}^{2+} \mathrm{Cbi}^{+}$in the presence of $\mathrm{CobA} / \mathrm{ATP}$ complex. 


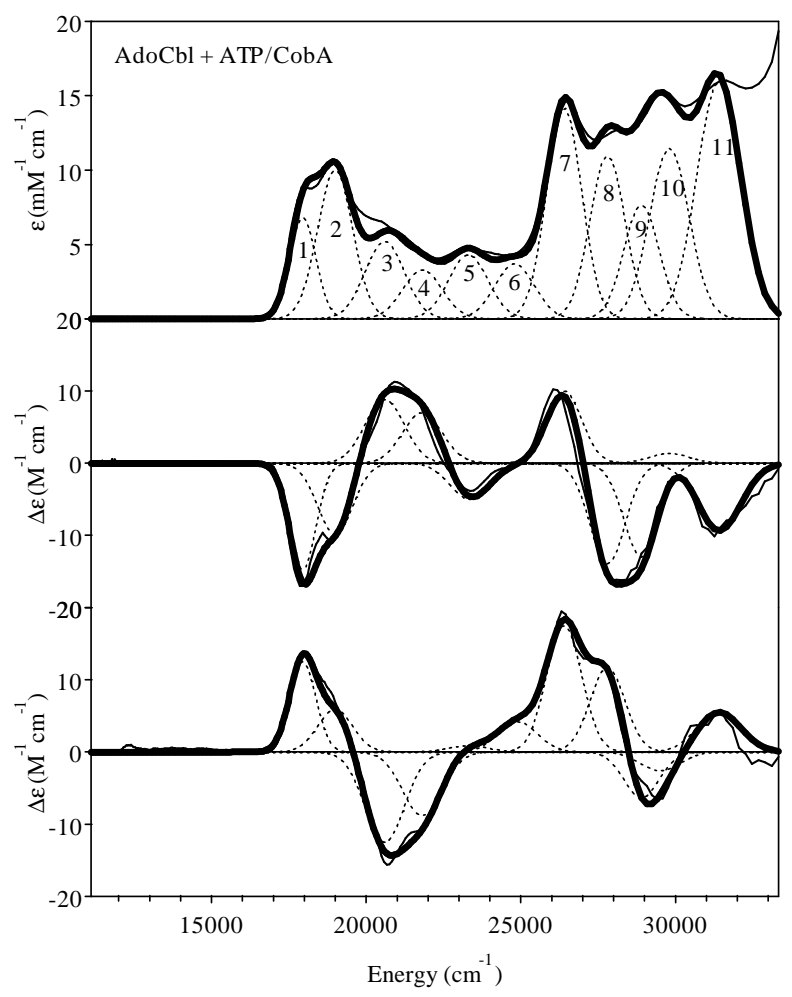

Figure S8. Solid lines: Absorption (top), CD (center), and 7 T MCD (bottom) spectra at $4.5 \mathrm{~K}$ of AdoCbl in the presence of ATP/CobA complex. Dotted lines: Gaussian deconvolutions of the experimental spectra. Bold lines: Sum of individual Gaussian bands. The fit parameters are given in Table S3. 
WEPR Simulation Parameters for free $\mathbf{C o}^{2+} \mathrm{Cbl}$. Figure 6A

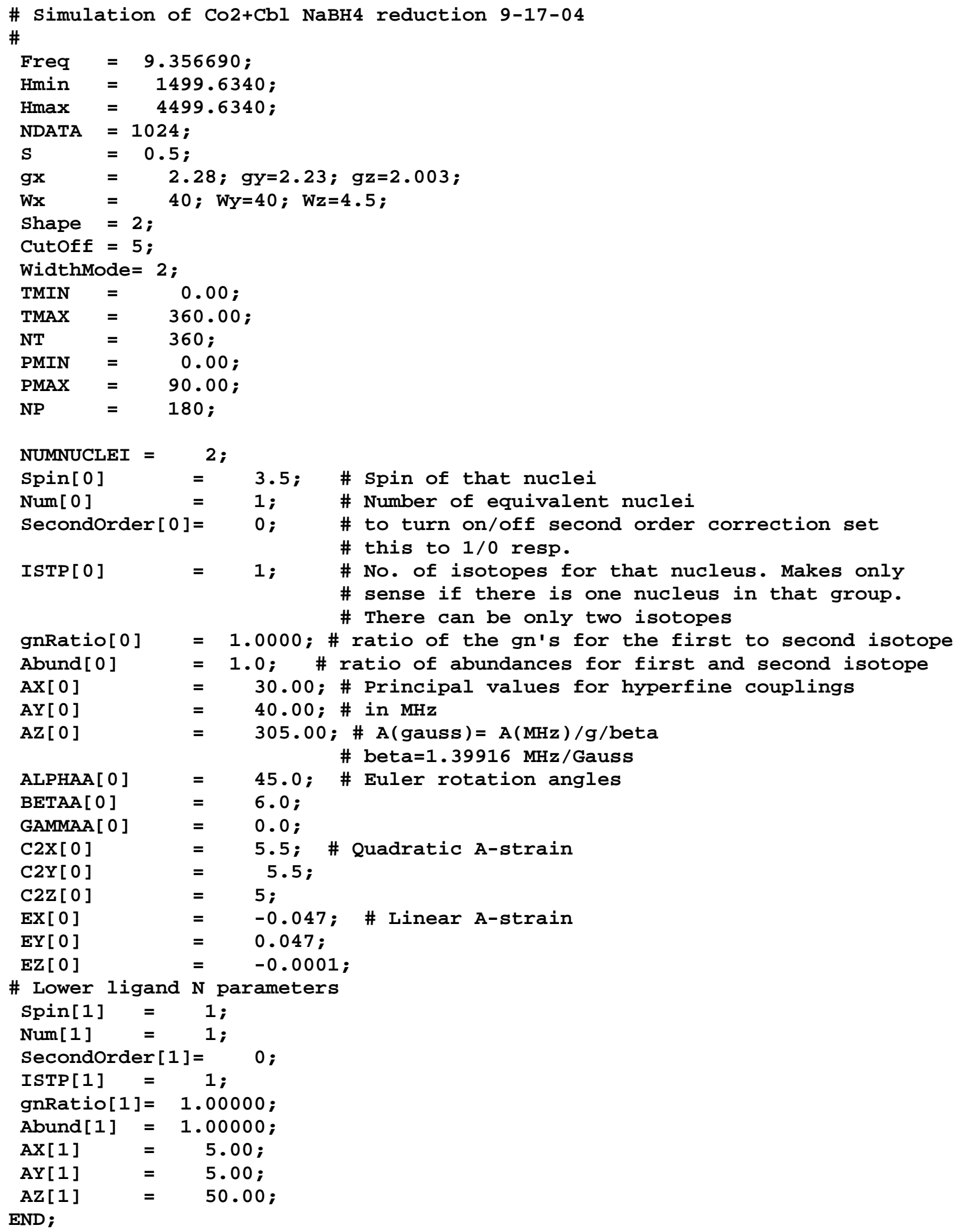


WEPR Simulation Parameters for free $\mathbf{C o}^{2+} \mathrm{Cbi}^{+}$. Figure 6D

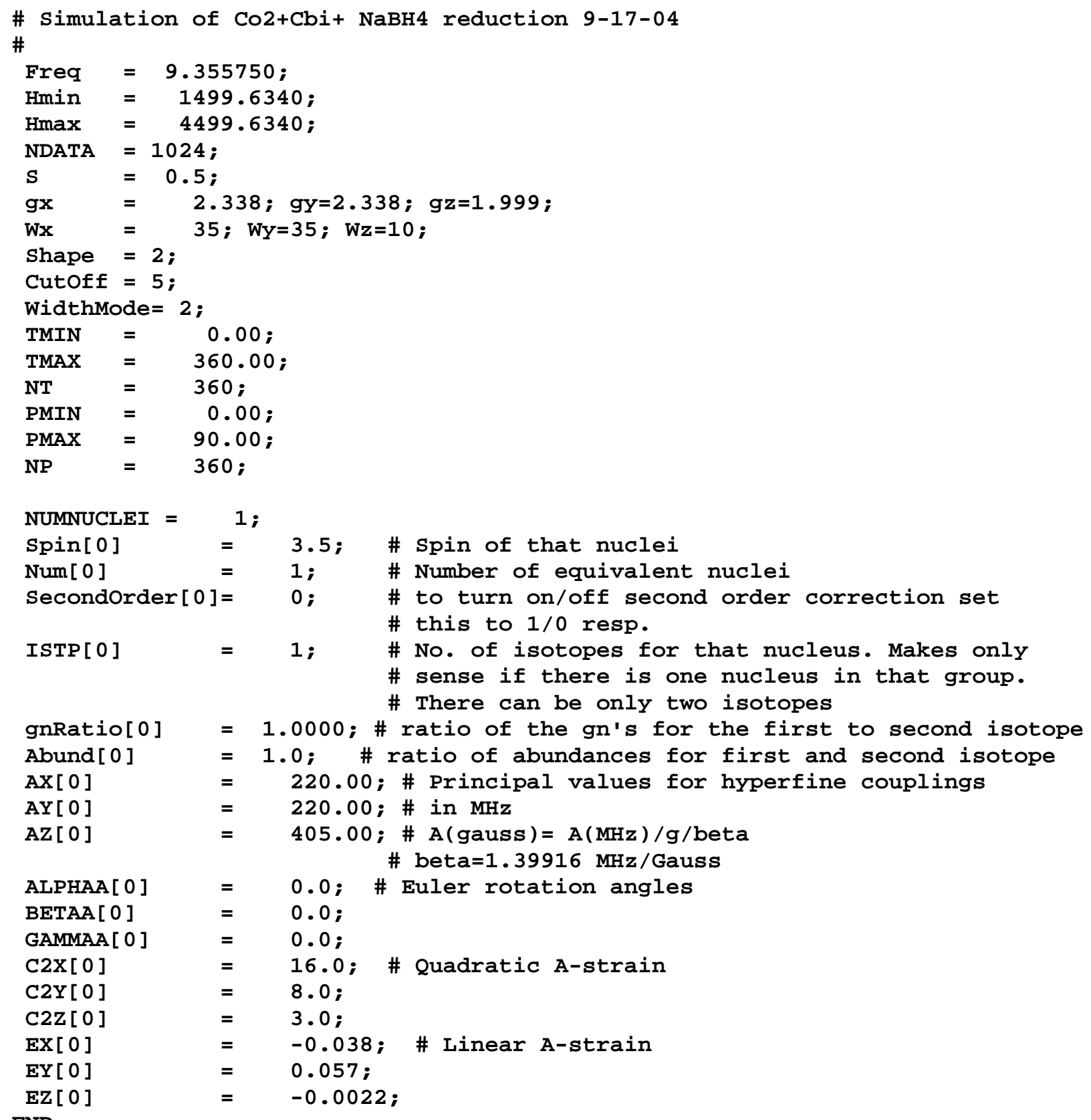


WEPR Simulation Parameters for $\mathrm{Co}^{2+} \mathrm{Cbi}^{+}$bound to $\mathrm{CobA} / \mathrm{ATP}$. Figure 6G

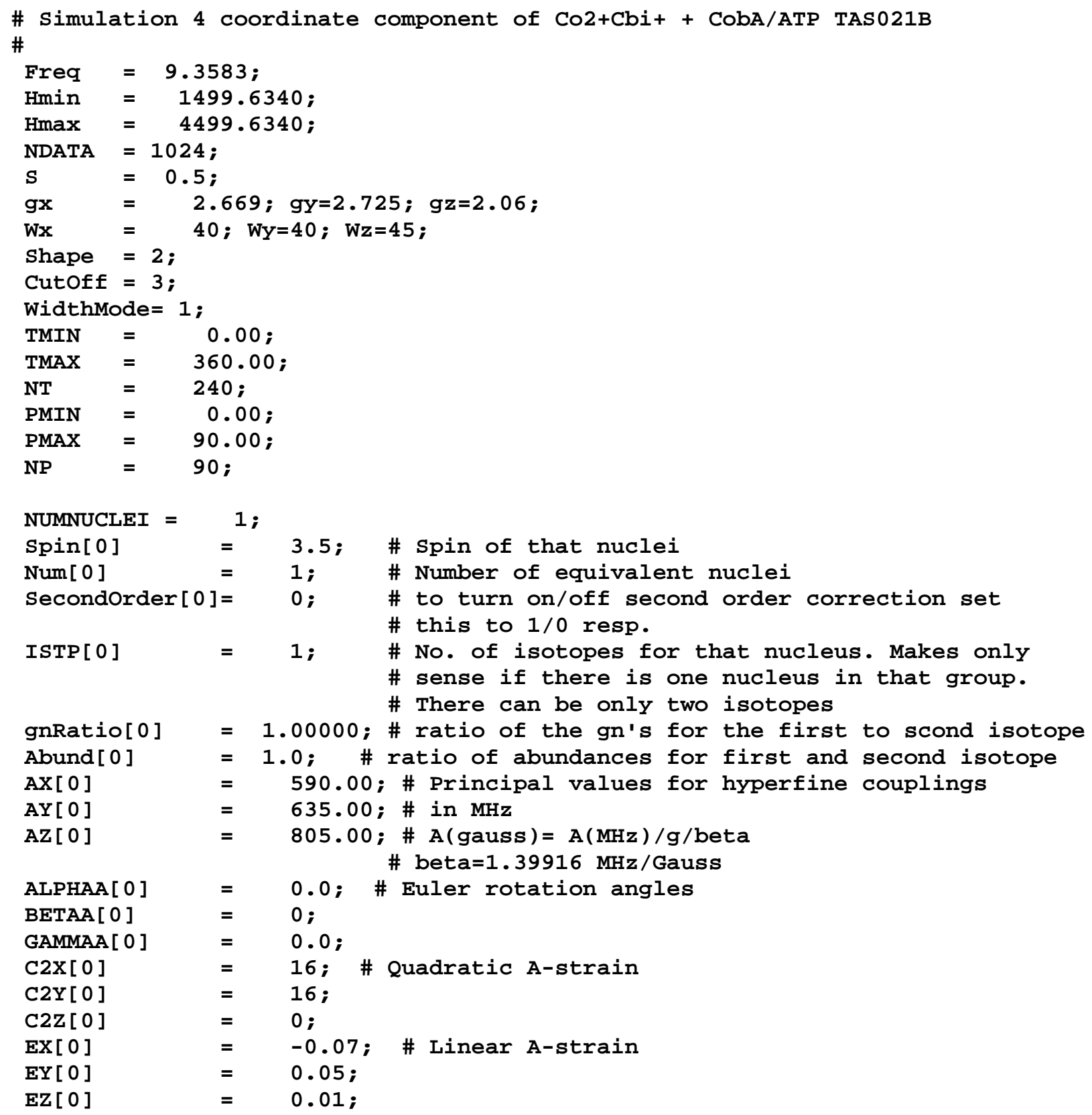

